8th International. Symposium on Electron Beam Ion Sources and Traps and their Applications, BNL, Upton, NY, November 6-8, 2000

\title{
Analysis of the Magnetic Field Measured by a Rotating Hall Probe in a Solenoid to Locate its Magnetic Axis
}

\author{
Ahovi Kponou, Alexander Pikin, Edward Beebe, James Alessi \\ Brookhaven National Laboratory \\ Upton, New York 11973-5000
}

\begin{abstract}
We have analyzed the motion of a Hall probe, which is rotated about an axis that is arbitrarily displaced and oriented with respect to the magnetic axis of a solenoid. We outline how the magnetic field measured by the rotating Hall probe can be calculated. We show how to compare theoretical results with actual measurements, to determine the displacement and orientation of the axis of rotation of the probe from the magnetic axis. If the center of rotation of the probe is known by surveying, the corresponding point on the magnetic axis of the solenoid can be located. This is applied to a solenoid that was built for BNL by Oxford Instruments.
\end{abstract}

\section{INTRODUCTION}

After a $5 \mathrm{~T}, 1 \mathrm{~m}$ long superconducting solenoid was delivered by Oxford Instruments, it was decided to do an independent measurement of the straightness of the magnetic axis. A common way to perform this measurement is to rotate a Hall probe at several points along the axis of the solenoid, recording measured field vs rotation angle at each axial stop. Information about the displacement of the axis of rotation from the magnetic axis is obtained by analyzing the measured data. In this paper, we derive from first principles the field measured by a rotating Hall probe, whose axis of rotation is arbitrarily displaced from and arbitrarily oriented to the magnetic axis of a solenoid. We use the results we obtain to analyze the measurements made for the Oxford Instruments solenoid. We are aware of the work by Stockli et. al., in which counterweights were used to straighten out the guide tube before making measurements[1]. Our analysis makes this unnecessary.

\section{THEORY}

Assume that the magnetic axis is along the $\mathrm{z}$-axis of the $\mathrm{x}-\mathrm{y}-\mathrm{z}$ rectangular coordinate system (Fig. 1), and that the axis of rotation of the probe is initially parallel to it, and displaced by some vector, $\boldsymbol{d} \boldsymbol{R}-$ (a) to (b) in Fig.1. For convenience, we take 
$d R$ to be perpendicular to the $z$-axis. ( $d R$ varies in magnitude and direction, along the axis of the magnet if the probe assembly moves in a tube which is not parallel to the $\mathrm{z}$ axis.) The tilt of the instantaneous axis of rotation of the probe, relative to the magnetic axis, can be reproduced by two Euler-type rotations. The first rotation is about the $X_{1}$-axis - by the angle $\theta_{x}-(\mathrm{b})$ to (c). It is represented by the matrix $R x$, and results in the $x^{\prime}-y^{\prime}-z^{\prime}$ coordinate system. The second rotation, about the new Y'-axis, by the angle $\theta_{y}-(\mathrm{c})$ to (d), is represented by $R y$ and results in the $\mathrm{x}^{\prime \prime}-\mathrm{y}^{\prime \prime}-\mathrm{z}$ " coordinate system. [2]:

$$
R x=\left[\begin{array}{ccc}
1 & 0 & 0 \\
0 & \cos \left(\theta_{x}\right) & \sin \left(\theta_{x}\right) \\
0 & -\sin \left(\theta_{x}\right) & \cos \left(\theta_{x}\right)
\end{array}\right] \quad R y=\left[\begin{array}{ccc}
\cos \left(\theta_{y}\right) & 0 & -\sin \left(\theta_{y}\right) \\
0 & 1 & 0 \\
\sin \left(\theta_{y}\right) & 0 & \cos \left(\theta_{y}\right)
\end{array}\right]
$$

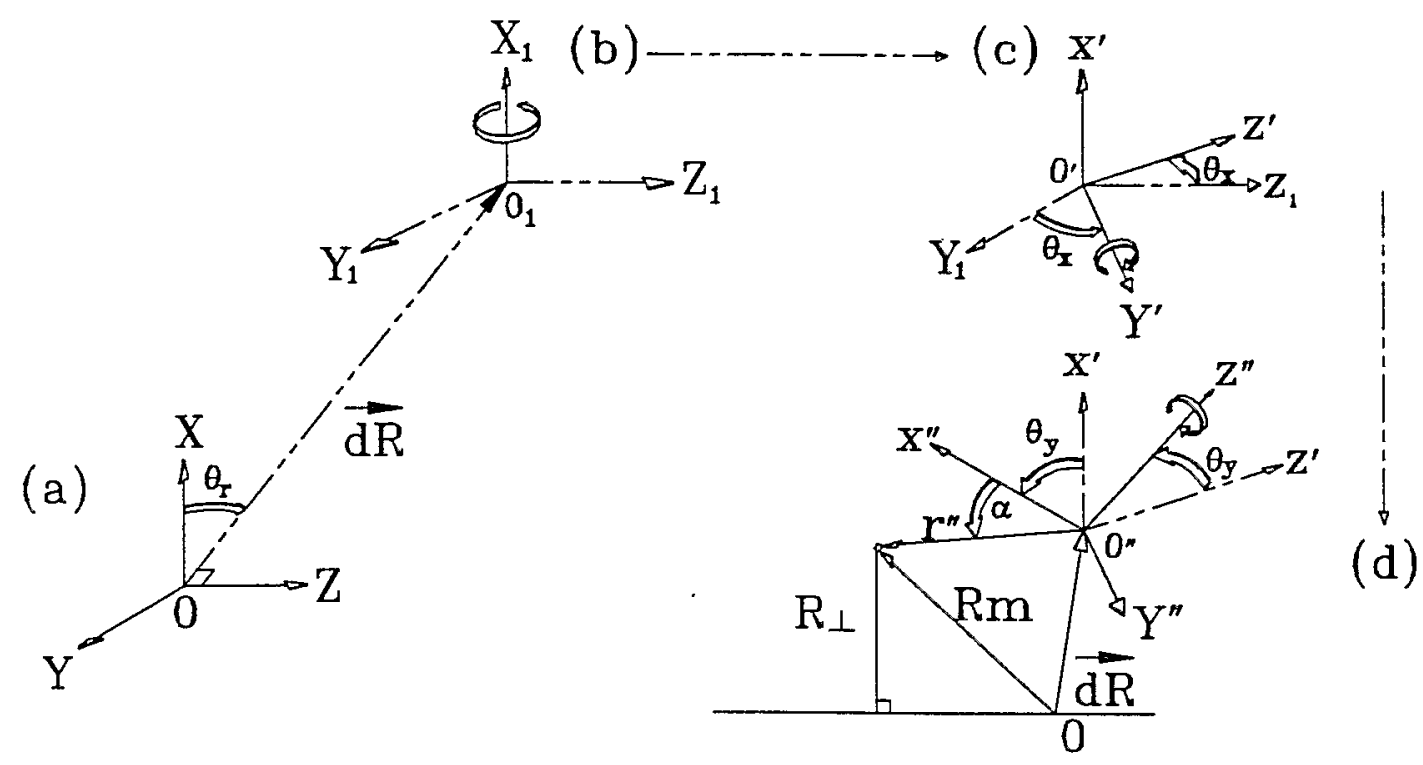

FIGURE 1. Generating an arbitrary displacement and orientation of the probe axis of rotation.

The resultant matrix is:

$$
R \equiv R y * R x \equiv\left[\begin{array}{ccc}
\cos \left(\theta_{y}\right) & \sin \left(\theta_{y}\right) \cdot \sin \left(\theta_{x}\right) & -\sin \left(\theta_{y}\right) \cdot \cos \left(\theta_{x}\right) \\
0 & \cos \left(\theta_{x}\right) & \sin \left(\theta_{x}\right) \\
\sin \left(\theta_{y}\right) & -\cos \left(\theta_{y}\right) \cdot \sin \left(\theta_{x}\right) & \cos \left(\theta_{y}\right) \cdot \cos \left(\theta_{x}\right)
\end{array}\right]
$$


The probe rotates in the $x^{\prime \prime}-y^{\prime \prime}$ plane, about $z^{\prime \prime}$. In this plane, its position is given by the polar coordinates $(a, \alpha)$, where $a$ is the radius of its circular path, and $\alpha$ is measured counterclockwise from the $\mathrm{x}^{\prime \prime}$ axis. In vector notation, the position of the probe is given by:

$$
\vec{r}^{\prime \prime} \equiv\left[\begin{array}{c}
a \cdot \cos (\alpha) \\
a \cdot \sin (\alpha) \\
0
\end{array}\right]
$$

The plane of the Hall probe is normal to $\vec{r}^{\prime \prime}$.

The matrices $R_{\mathrm{x}}, R_{y}$ (and $R$ ) are orthogonal, hence $R^{-1}$ is equal to its transpose, $R^{T}$. In the $\mathrm{x}-\mathrm{y}-\mathrm{z}$ frame of reference, i.e. the original frame, the radius vector of the probe is given by:

$$
\vec{r}=R^{T} * \vec{r}^{\prime \prime} \equiv a \cdot\left[\begin{array}{c}
\cos \left(\theta_{y}\right) \cdot \cos (\alpha) \\
\sin \left(\theta_{x}\right) \cdot \sin \left(\theta_{y}\right) \cdot \cos (\alpha)+\cos \left(\theta_{x}\right) \cdot \sin (\alpha) \\
-\cos \left(\theta_{x}\right) \cdot \sin \left(\theta_{y}\right) \cdot \cos (\alpha)+\sin \left(\theta_{x}\right) \cdot \sin (\alpha)
\end{array}\right]
$$

and it has the unit vector:

$$
\vec{r}_{U}=\frac{1}{|\vec{r}|} \cdot \vec{r}=\frac{1}{a} \cdot \vec{r}
$$

The displacement vector of the center of rotation, $O^{\prime}$, of the probe from the magnetic axis, $O$ is given by:

$$
d \vec{R} \equiv\left[\begin{array}{c}
d R \cdot \cos \left(\theta_{r}\right) \\
d R \cdot \sin \left(\theta_{r}\right) \\
0
\end{array}\right]
$$

The position vector of the probe from the point $O$ on the magnetic axis is given by:

$$
\vec{R}_{m}=\vec{r}+d \vec{R}
$$


The $\mathrm{x}$ - and $\mathrm{y}$-components are:

$$
\begin{aligned}
& R_{m x}=a \cdot \cos \left(\theta_{y}\right) \cdot \cos (\alpha)+d R \cdot \cos \left(\theta_{r}\right) \\
& R_{m y}=a \cdot\left(\sin \left(\theta_{x}\right) \cdot \sin \left(\theta_{y}\right) \cdot \cos (\alpha)+\cos \left(\theta_{x}\right) \cdot \sin (\alpha)\right)+d R \cdot \sin \left(\theta_{r}\right)
\end{aligned}
$$

Let

$$
\vec{R}_{\perp}=\left[\begin{array}{c}
R_{m x} \\
R_{m y} \\
0
\end{array}\right] \equiv\left[\begin{array}{c}
R_{\perp_{-} x} \\
R_{\perp_{-} y} \\
R_{\perp_{-} z}
\end{array}\right]
$$

be the perpendicular displacement vector of the probe from the magnetic axis. The radial magnetic field at the probe, $B_{r}$, will be along $\vec{R}_{\perp}$, whose unit vector is given by:

$$
\vec{R}_{\perp \_U}=\frac{1}{\left|\vec{R}_{\perp}\right|} \cdot \vec{R}_{\perp}
$$

Hence:

$$
B_{r}=B_{r} \cdot \vec{R}_{\perp_{-} U}
$$

The total magnetic field, $B$, is:

$$
\vec{B}=B_{r} \cdot \vec{R}_{\perp_{-} U}+B_{z} \cdot \vec{k}
$$

$B_{z}$ and $\boldsymbol{k}$ are, respectively, the longitudinal field component and the unit vector in the $z$-direction. POISSON calculations based on the design model of the magnet were used to obtain field maps. These maps allowed us to find polynomial expressions in $\vec{R}_{\perp}$ and $\mathrm{z}$ for both $B_{r}$ and $B_{z}$, at the probe position, which is given by $\mathrm{z}$ and $\vec{R}_{\perp}$. (The angular dependence on the angle $\alpha$ (see Fig. 1) shows up in the variation of $\vec{R}_{\perp}, z$ is measured from the mid-point of the magnet.)

The field normal to the Hall probe, the measurement, is given by the scalar product of $\boldsymbol{B}$ and the unit vector normal to the probe, i.e.

$$
B_{N}=\vec{B} \bullet \vec{r}_{U}
$$

$B_{N}$ is to be fitted to the measured fields using $\theta_{x}, \theta_{y}, \theta_{n}$ and $d R$ as fitting parameters. 
We show curves of $B_{N} v s$ rotation angle, $\alpha$, for several combinations of the fitting parameters; at the mid-plane in Fig. 2, and at $\mathrm{z}=-50 \mathrm{~cm}$, in Fig. 3. The legend entries give $\theta_{x}, \theta_{y}$, and $\theta_{r}$ in milliradians, and $d R$, in centimeters, in this order.

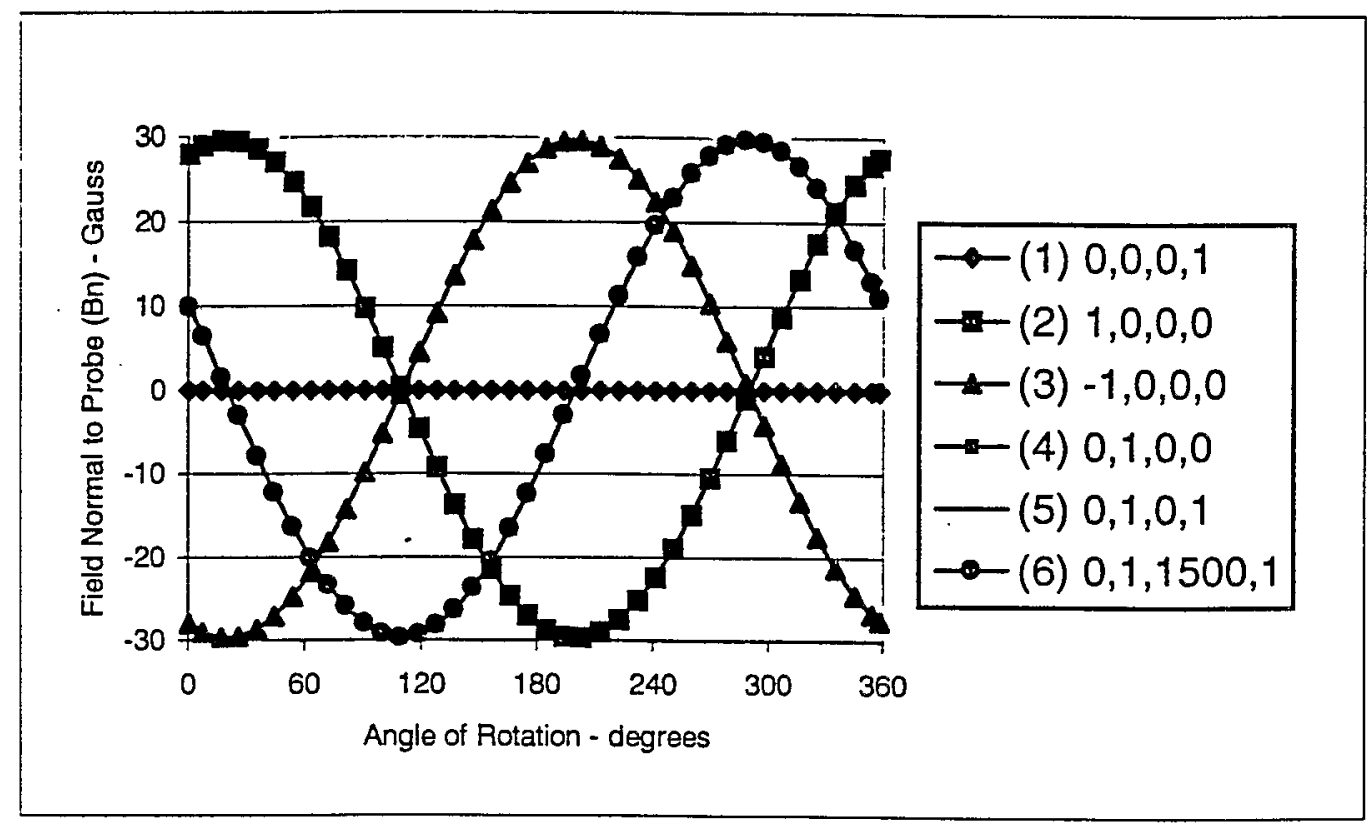

FIGURE 2. Theoretical curves at the mid-plane of the solenoid. Curves (4), (5) coincide with (6) for the vertical scale used. The quartet of numbers are $\theta_{x}, \theta_{y}, \theta_{r}$, in milliradians, and $d R$, in centimeters.

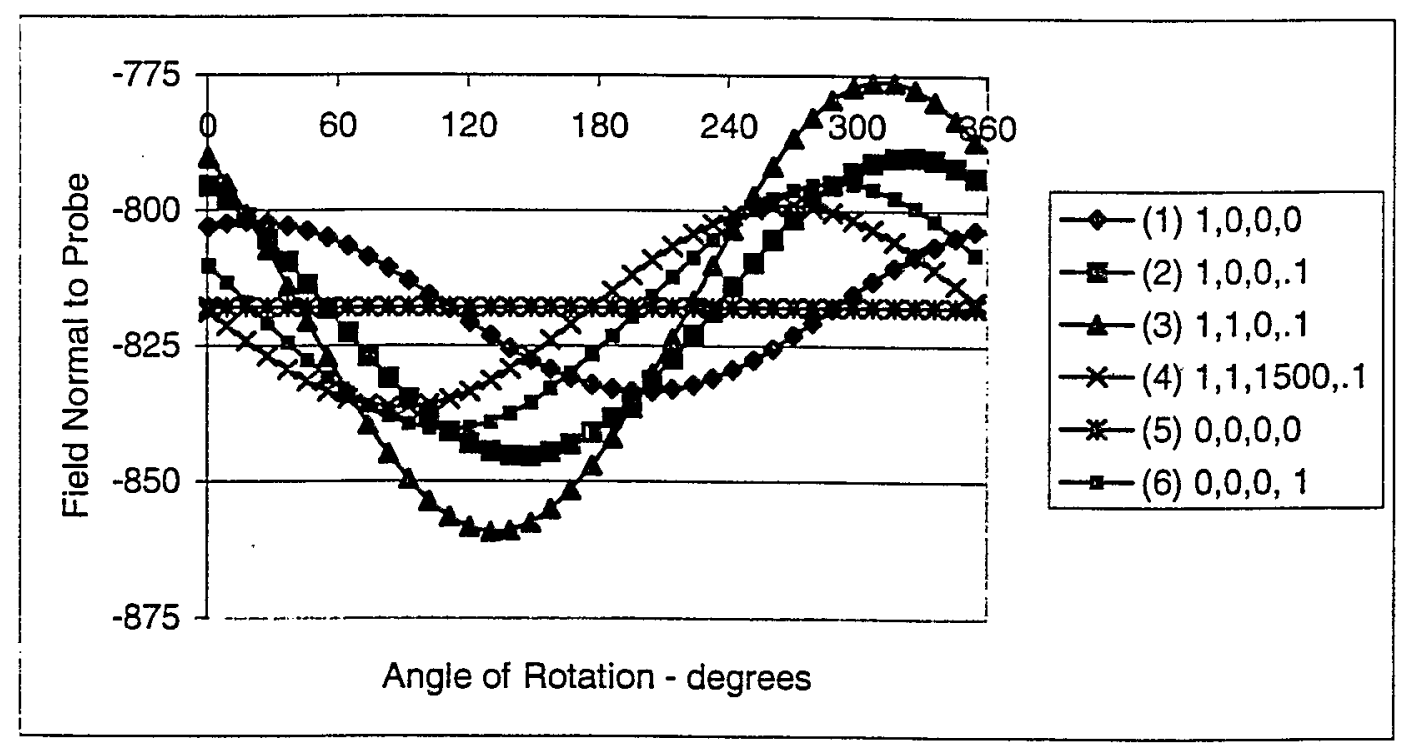

FIGURE 3. Theoretical curves at $\mathrm{z}=-50 \mathrm{~cm}$, near where the radial component is a maximum 


\section{MEASUREMENTS}

The layout of the measurement setup is shown in Fig. 4. The drive shaft was connected to the probe assembly by a universal joint at one end, and to a PC controlled motor drive assembly at the other. This provided longitudinal and rotational motion of the probe. The probe output was amplified, sampled, and saved by another PC running LABVIEW. The probe carriage assembly moved in a stainless steel tube, which was supported by four adjusting screws at $\pm 71 \mathrm{~cm}$ from the mid-plane of the solenoid.

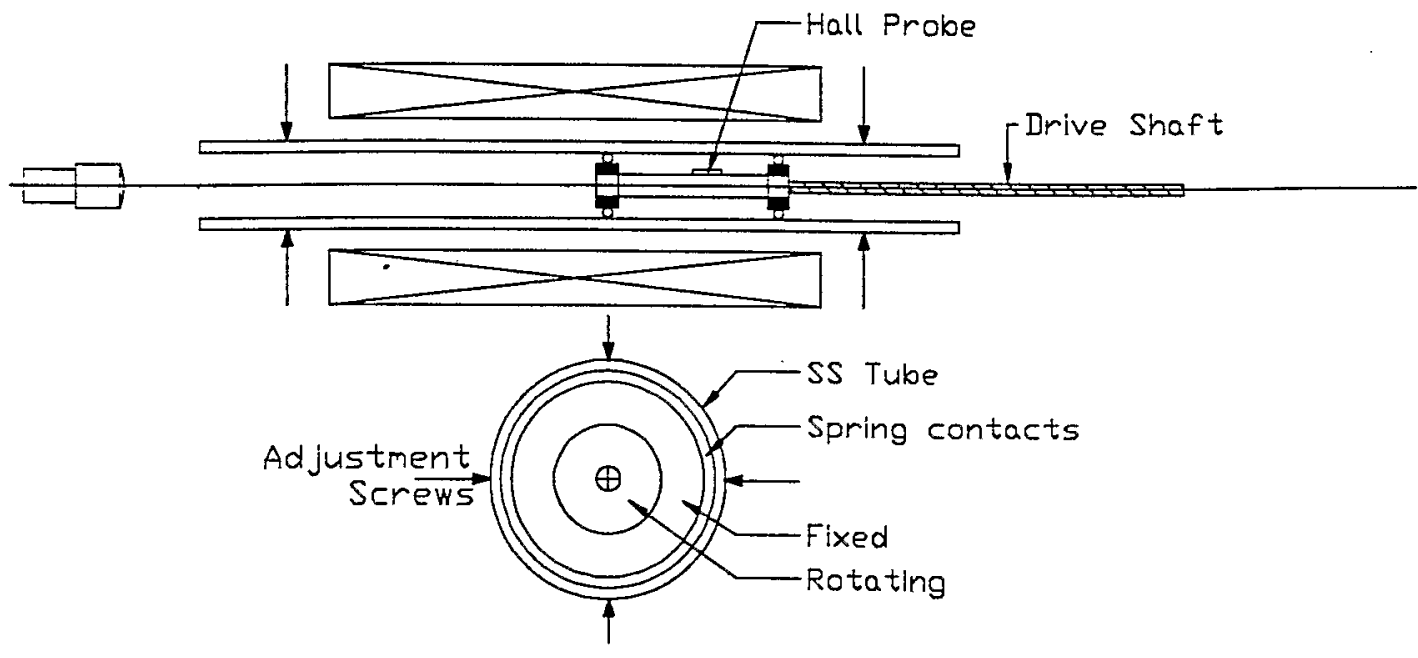

FIGURE 4. A layout of the measurement setup. The lower drawing is a section through one of the tube supports at $\pm 71 \mathrm{~cm}$ from the mid-plane of the solenoid.

\section{Experimental Procedure.}

With the probe positioned at the adjusting screws, the tube position was adjusted to bring the center of rotation of the probe into coincidence with the magnetic axis. Ideally, when this is the case, the probe output is constant. In practice, it may not be, because the axis of rotation could be tilted. The adjustment we made was to minimize the sinusoids at these locations as the probe was rotated. After this adjustment, then starting at $+65 \mathrm{~cm}$ from the mid-plane, the probe was moved in steps of $-5 \mathrm{~cm}$. At each step, the probe was rotated through $360^{\circ}$, and the center of the front of the probe carriage surveyed. (The longitudinal position of the probe was calculated from the geometry of the probe assembly.) The survey results, which essentially give the profile of the stainless steel tube, are shown in Fig. 5. The results of regression analysis of the data for both planes are displayed. Using the equations in Fig. 5, and the geometry of the probe carriage, the transverse position of the center of rotation of the probe was determined at each step. 
As the probe made a complete rotation at each longitudinal position, its output was sampled at equally spaced intervals, digitized, and stored. At the end of the rotation, the data were saved in ASCII format for later use in a spreadsheet, where the conversion to magnetic field and a preliminary fit to the data were made.

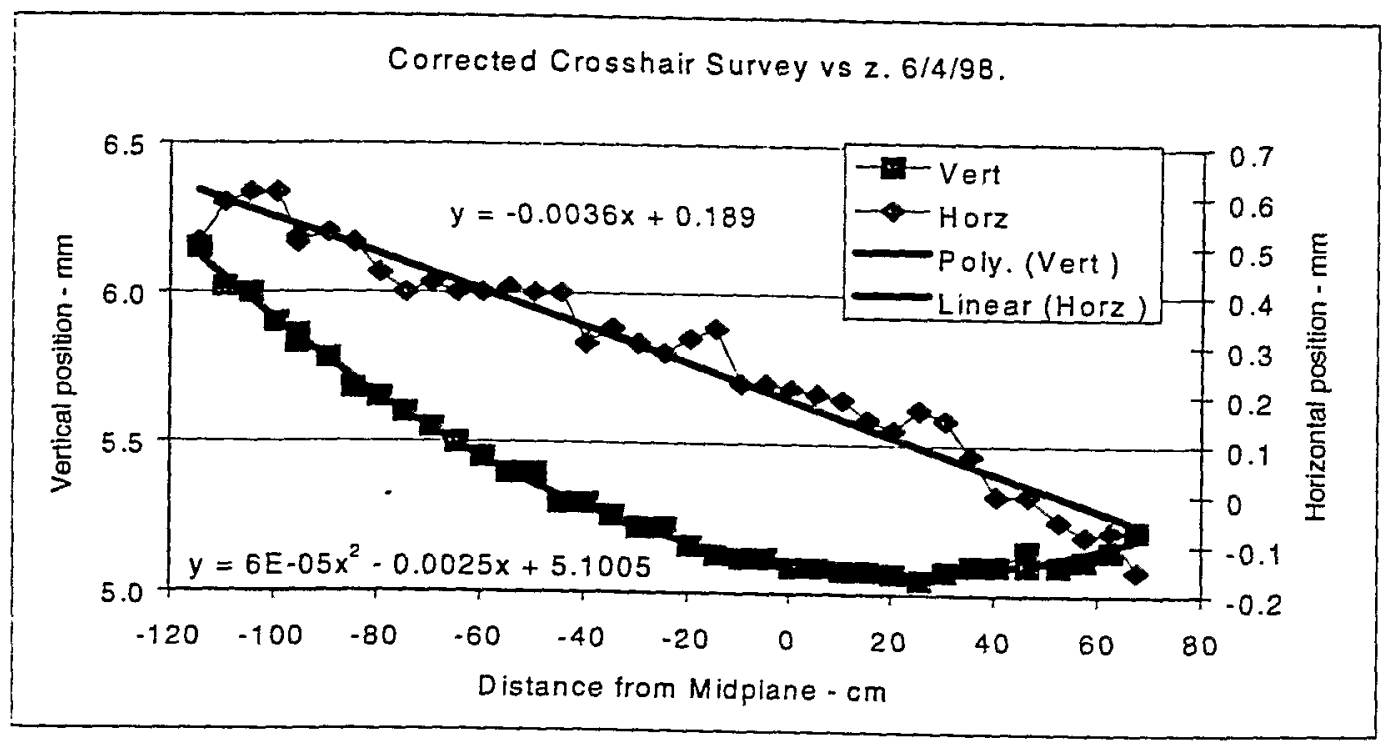

FIGURE 5. Path of the center of rotation of the Hall probe as it moved through the solenoid. It clearly shows the vertical sag in the stainless steel guide tube. The solid lines are linear and quadratic fits.

The parameters from the preliminary fit were then used to begin the search with a FORTRAN program. It was found necessary to introduce a fifth fitting parameter, $B_{\text {off }}$, in order to obtain good fits. A possible contribution to $B_{\text {off }}$ is discussed in [3], and has to do with using a Hall probe in a strong inhomogeneous field, in an orientation in which there is a field component parallel to the plane of the probe, but not along one of its major axes. Two data sets, and the theoretical fits to them, are shown in Figs. $6 \mathrm{a}$ and $6 \mathrm{~b}$. Although, they were taken at equidistant positions from the mid-plane of the solenoid, there is a marked difference in the quality of the data. We believe this was due to strains developed in the signal leads of the Hall probe as it was rotated, which we could not eliminate in the time available to make the measurements.

From the analyses of the rotation data - Fig. 6, and the survey data - Fig. 2, points on the magnetic axis were found. These are plotted in Fig. 7. The data between $+/-35 \mathrm{~cm}$ were not used because of their poor quality, due to the problem with signal leads discussed earlier and to the smaller radial fields near the mid-plane of the solenoid. [The solenoid was designed to produce a very uniform field over half of its length of $1 \mathrm{~m}$.] It is not clear why the points show more variations on one side of the solenoid than on the other. 


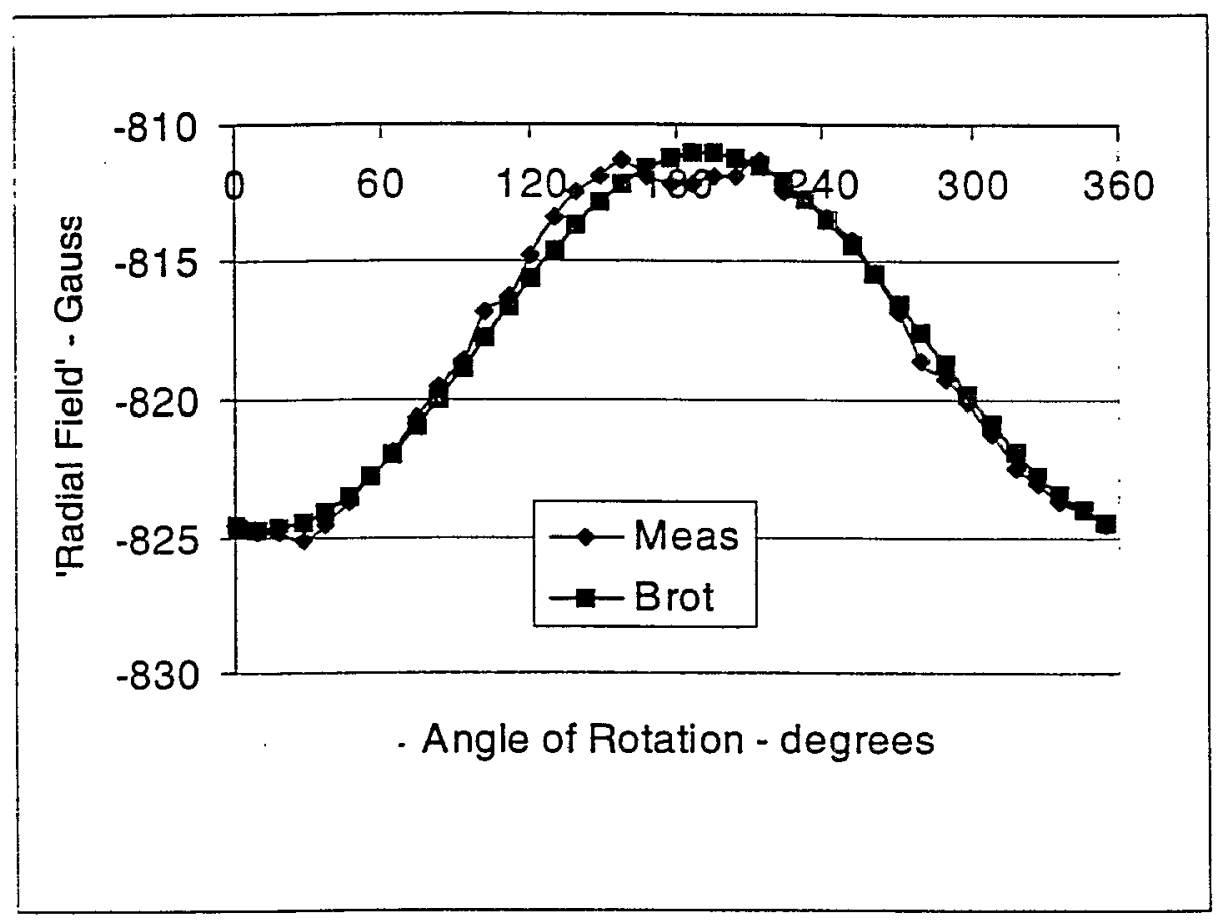

FIGURE $6 a .-50 \mathrm{~cm}$ from mid-plane of the solenoid. Fitted values for $\mathrm{dR}, \theta \mathrm{x}, \theta \mathrm{y}, \& \mathrm{r}$, and $\mathrm{B}_{\mathrm{off}}$ are, respectively: $0.2 \mathrm{~mm},-0.84,0.55,2600 \mathrm{mrad}$, and $-45.1 \mathrm{G}$.

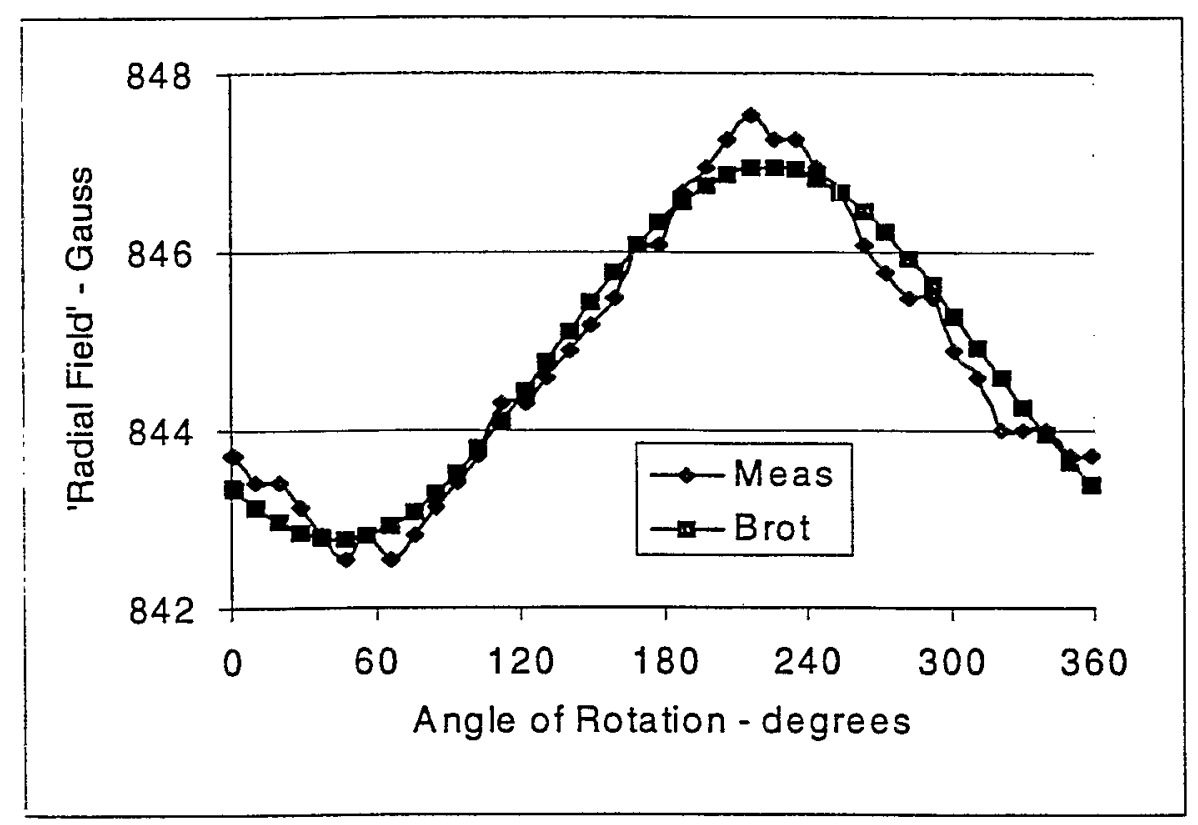

FIGURE $6 \mathrm{~b} .50 \mathrm{~cm}$ from mid-plane of the solenoid. Fitted values for $d R, \theta x, \theta y, \& \theta r$, and $B_{\text {off }}$ are, respectively: $0.04 \mathrm{~mm},-0.2,0.1,-1930 \mathrm{mrad}$, and $72.1 \mathrm{G}$. 


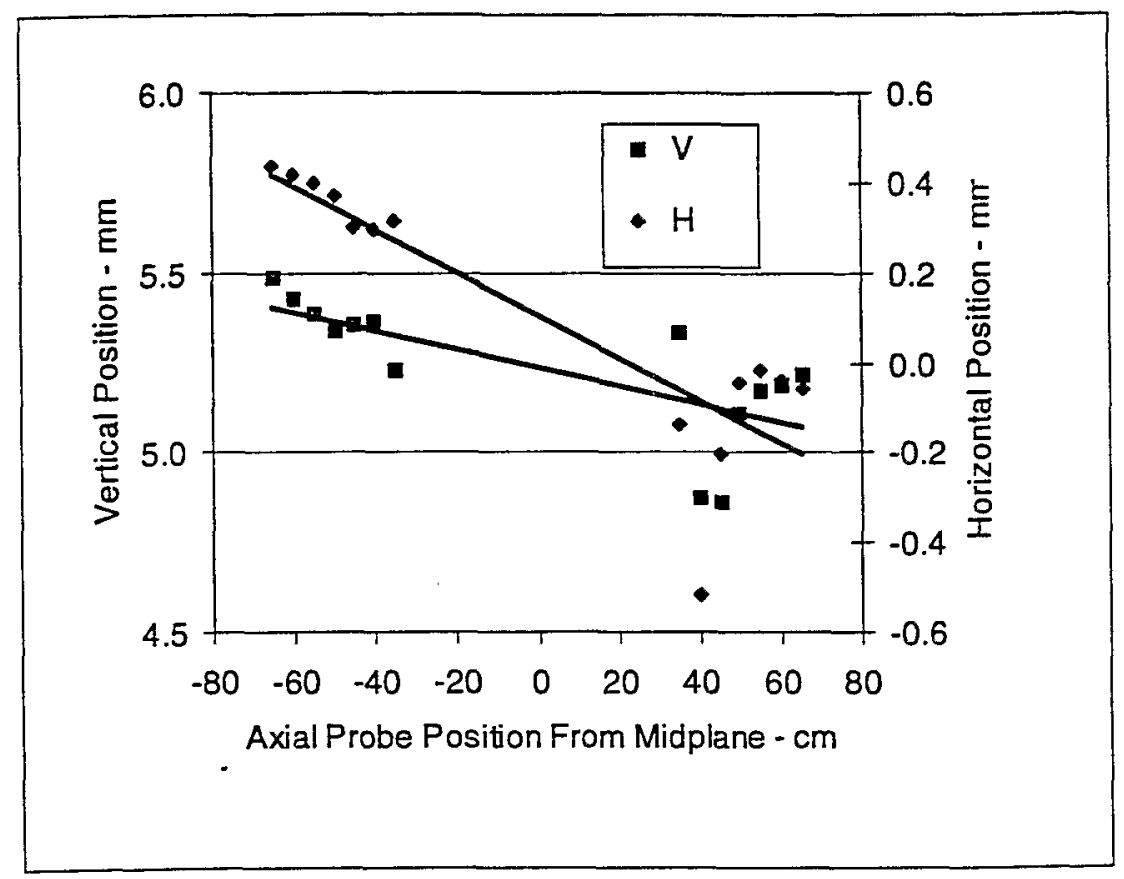

FIGURE 7. Points on magnetic axis determined from fits to probe rotation data and surveying. The straight lines are linear fits to the data. The positions are readings from the scale of the transit.

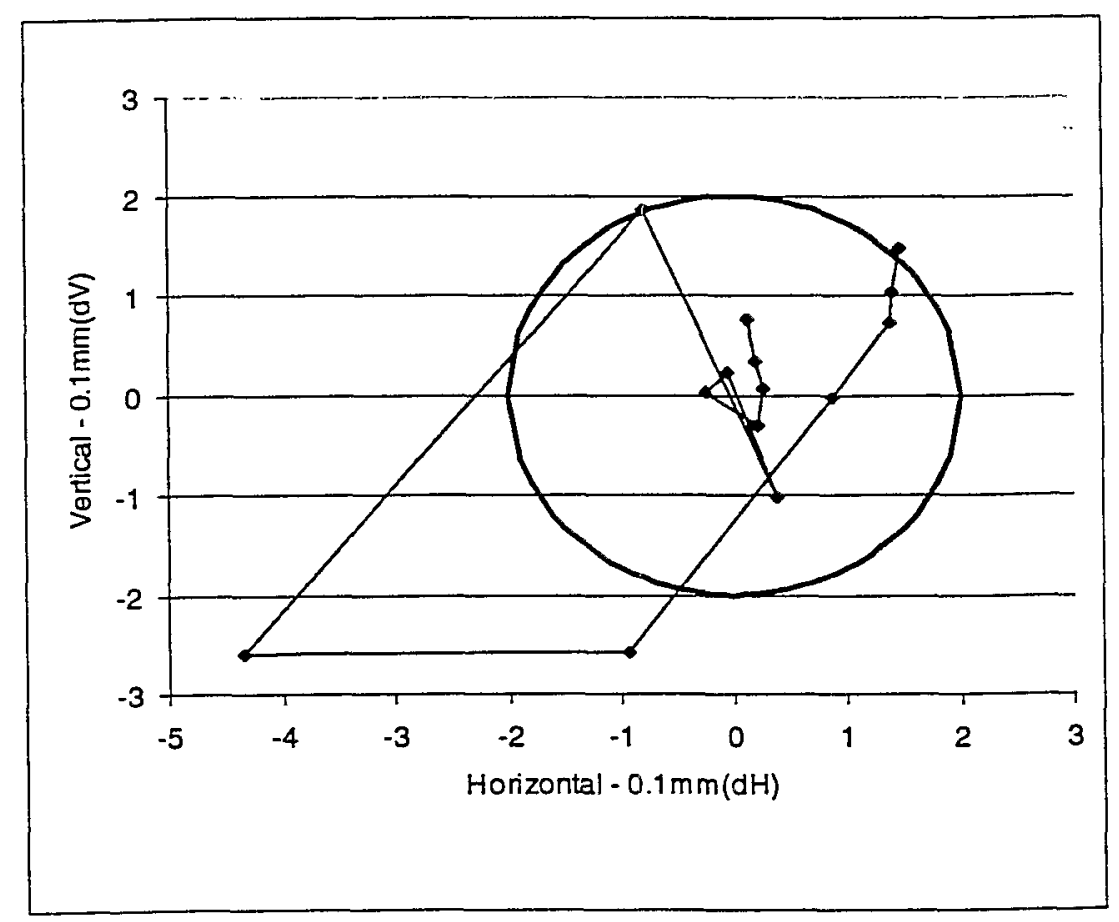

FIGURE 8. Scatter of measured magnetic axis points about the fitted axis. All but two points lie within a circle of radius $0.2 \mathrm{~mm}$. 
The deviations of the points from the fitted lines in Figure 7 are plotted in Figure 8. A circle of $0.2 \mathrm{~mm}$ radius, representing the design specification for the straightness of the magnetic axis, is also drawn in the figure. The two obviously "bad" points were obtained for the probe at 40 and $45 \mathrm{~cm}$. In general, the measurements on the negative side of the mid-plane show less scatter than those on the positive side, for reasons that might have had to do with strain of the signal leads.

\section{Observations and Conclusions}

We have successfully analyzed one type of motion of a flat Hall probe in a magnetic field, and showed how to use it to determine the straightness of the magnetic axis of a solenoid. The measured curves were not always smooth. Their jaggedness was partly due to strains in the signal leads of the Hall probe as the latter was rotated. Time did not allow us to fix this problem.

\section{ACKNOWLEDGEMENTS}

This work was performed under the auspices of the U.S. Department of Energy.

Our special thanks to Walter Hensel, Daniel McCafferty, and Werner Tramm for making the experiment work, and to the BNL Magnet Measurements Group, for the loan of a computer controlled motor drive unit.

\section{REFERENCES}

1. A. Hershcovitch et al., BNL Informal Report \# BNL-60509 (6/3/94). See Appendix.

2. See, for example, G. Arfken, Mathematical Methods for Physicists, $3^{\text {rd }}$ Edition, Academic Press, 1985. Ch. 4.

3. M Turin, N.I.M., 91 621-625 (1971). 